\title{
Inhibition of protease of Novel Corona Virus by Designed Noscapines: Molecular docking and ADMET studies
}

Vijay Kumar Vishvakarma, ${ }^{1}$ Kamlesh Kumari, ${ }^{2}$ Prashant Singh ${ }^{1, *}$

${ }^{1}$ Department of Chemistry, Atma Ram Sanatan Dharma College, University of Delhi, New

Delhi, India ; ${ }^{2}$ Department of Zoology, Deen Dayal Upadhyaya College, University of Delhi, New Delhi, India

*Corresponding Author; Email: psingh@arsd.du.ac.in

\begin{abstract}
:
Nowadays, many people were dying due to infectious coronavirus diseases (COVID-19). It belongs to the betacoronavirus family and also known as SARS-CoV-2. However, COVID19 is a new form that has some basic difference in the genome which makes it more lethal and infectious. In transmitted in human in late December 2019 and it infected about 20 million till date. Its genome is composed of positive-sense single-stranded RNA, which encodes for the poly-protein. This poly-protein further cleaved into various components of the virus to make the numerous copy of the virus. There are many more similarities in their genome among the SARS-CoV-2, SARS-CoV, MERS-CoV. However, protease proteins are responsible for the cleavage and hence, COVID-19 main protease is a prime therapeutic target. To date, no medicine/ vaccine can fully cure their infection. To inhibit the activity of protease of COVID-19, molecular docking and ADMET studies of 116 noscapine derivatives were performed and the result was compared with 14 reputed antiviral drugs including chloroquine and hydroxychloroquine. The molecular docking result indicates a better binding in comparison of 14 reputed drugs. Further, the top six noscapines was taken into consideration for the pose analysis and ADMET studies. Finally, the top six noscapine was refined by ADMET properties to get the most potent one.
\end{abstract}

Keywords: COVID-19; protease of SARS-CoV-2; Noscapine; Inhibition; Molecular Docking; ADMET 


\section{Introduction}

Novel COVID-19 (Coronavirus Disease) originates from Wuhan, China in late December 2019.(Alexander \& Qato, 2020; Bodas \& Peleg, 2020) It is a new type of human coronavirus and spreading very fast in a contagious manner. As time passes it spread to the whole world and acquire the pandemic nature.(Auerbach \& Miller, 2020; Bayefsky, Bartz, \& Watson, 2020; Campbell \& Kahwash, 2020) About 20 million confirmed cases till date from the whole world.(https://www.who.int/emergencies/diseases/novel-coronavirus-2019) COVID19 and SARS-CoV-2 both belong to betacoronavirus genus. Its genome is positive singlestranded RNA.(Phua et al., 2020) It is associated with the many diseases starting from the respiratory infection to severe pneumonia or acute respiratory distress syndrome (ARDS).(Mather et al., 2020; Peloso, Moeckli, Oldani, Triponez, \& Toso, 2020) Sometimes patient get recovered due to its immune response but in the case of pre-existing clinical complexes leads to death. It mainly transmitted by the droplet of the infected peoples.(Clarke, Stephens, Liao, Byrne, \& Gregory, 2020; Hooli \& King, 2020) Due to ARDS, finally the patient suffers from the multi-organ failure (MOF) and resulted in death.(Phua et al., 2020; Vincent \& Taccone, 2020) However, the whole structure of the COVID-19 is not reported to date but in most of the viruses, proteases are mainly responsible for the catalytic infectious activity.(Aydemir \& Ulusu, 2020; Bayefsky et al., 2020) Molecular docking is a computational technique used to find the interaction between the drug and the amino acid of the proteins.(Azam \& Jupudi, 2019) It gives atomic level interaction between them. A huge number of ligand library can be screened by the binding energy value obtained by the molecular docking.(V. K. S. Vishvakarma, N.; Reetu; Kumari, K.; Patel R.; Singh, P., 2019; Vora et al., 2019)

Noscapine is an alkaloid derived from the Papaver somniferum.(Kumar et al., 2019a) It has an antitussive and anti-cancerous property and non-addictive, which makes it different from the other opioid products.(Alijanvand et al., 2020; Altinoz et al., 2019; Chandra et al., 2012; Kumar, Kumari, Jayaraj, Kumar, Kumar et al., 2020; Kumar et al., 2019b; H. Singh et al., 2013; Singh \& Chandra, 2012; Singh et al., 2019; Singh, Singh, Chandra, Dass, \& Chandra, 2013; Singh et al., 2017; V. K. Vishvakarma, Kumari, \& Singh, 2020) There is too much clinical utility of the opium-derived drugs that's why many researchers focused on it. It is found in two isomeric forms viz., erythro-noscapine and threo-noscapine.(Kocak, Kocak, Ozturk, Tekin, \& Vatansev, 2020) The stability of erythro-noscapines makes it more useful than threo-nocapine.(Kumar et al., 2019a; Muthiah et al., 2019) Herein, a total of 116 noscapine derivatives (pre-existing and virtual) were optimized and studied their potential 
against the protease of SARS-CoV-2 using molecular docking. Further, absorption, distribution, metabolism, excretion and toxicity (ADMET) properties of the potential inhibitors were determined to further refine the results.

\section{Experimental}

\subsection{Designing of molecules and protease for molecular docking}

\subsubsection{Designing of molecules}

Noscapine was chosen due to its potential in different biological potency and then a library of the molecules was created virtually. In the present work, a total 115 derivatives of noscapine ware created by substituting at the 9' position as in Table 1.(Kumar et al., 2019a)

Table 1: Libraries of the molecules based on noscapine

\begin{tabular}{|c|c|c|c|c|c|c|c|c|c|}
\hline \multirow{2}{*}{$\begin{array}{r}\text { Parent compound } \\
\mathrm{R}\end{array}$} & \multicolumn{4}{|c|}{ Alkyl Group (R) } & \multirow[t]{2}{*}{ Parent compound } & \multicolumn{4}{|c|}{ Alkyl Group (R) } \\
\hline & 1 & $-\mathrm{H}$ & 11 & $-\mathrm{COOH}$ & & 21 & $-\mathrm{H}$ & 31 & $-\mathrm{COOH}$ \\
\hline \multirow{9}{*}{$R$} & 2 & $-\mathrm{CH}_{2} \mathrm{OH}$ & 12 & $-\mathrm{CHO}$ & & 22 & $-\mathrm{CH}_{2} \mathrm{OH}$ & 32 & $-\mathrm{CHO}$ \\
\hline & 3 & $-\mathrm{CH}_{2} \mathrm{Br}$ & 13 & $-\mathrm{COCH}_{3}$ & & 23 & $-\mathrm{CH}_{2} \mathrm{Br}$ & 33 & $-\mathrm{COCH}_{3}$ \\
\hline & 4 & $-\mathrm{CH}_{2} \mathrm{Cl}$ & 14 & $-\mathrm{CH}=\mathrm{CH}_{2}$ & & 24 & $-\mathrm{CH}_{2} \mathrm{Cl}$ & 34 & $-\mathrm{CH}=\mathrm{CH}_{2}$ \\
\hline & 5 & $-\mathrm{NO}_{2}$ & 15 & $-\mathrm{CH}_{3}$ & & 25 & $-\mathrm{NO}_{2}$ & 35 & $-\mathrm{CH}_{3}$ \\
\hline & 6 & $-\mathrm{NH}_{2}$ & 16 & $-\mathrm{OCH}_{3}$ & & 26 & $-\mathrm{NH}_{2}$ & 36 & $-\mathrm{OCH}_{3}$ \\
\hline & 7 & $-\mathrm{Cl}$ & 17 & $-\mathrm{OCH}_{2} \mathrm{CH}_{3}$ & & 27 & $-\mathrm{Cl}$ & 37 & $-\mathrm{OCH}_{2} \mathrm{CH}_{3}$ \\
\hline & 8 & $-\mathrm{Br}$ & 18 & $-\mathrm{OH}$ & & 28 & $-\mathrm{Br}$ & 38 & $-\mathrm{OH}$ \\
\hline & 9 & -NHAc & 19 & $-\mathrm{COBr}$ & & 29 & -NHAc & 39 & $-\mathrm{COBr}$ \\
\hline & 10 & $-\mathrm{COCl}$ & 20 & $-\mathrm{CN}$ & & 30 & $-\mathrm{COCl}$ & 40 & $-\mathrm{CN}$ \\
\hline Parent compound & \multicolumn{4}{|c|}{ Alkyl Group (R) } & Parent compound & \multicolumn{4}{|c|}{ Alkyl Group (R) } \\
\hline \multirow{10}{*}{$0_{1}^{\prime} \mathrm{O}-0$} & 41 & $-\mathrm{CH}_{2} \mathrm{OH}$ & 51 & $-\mathrm{CHO}$ & \multirow{10}{*}{0} & 60 & $-\mathrm{CH}_{2} \mathrm{OH}$ & 70 & $-\mathrm{CHO}$ \\
\hline & 42 & $-\mathrm{CH}_{2} \mathrm{Br}$ & 52 & $-\mathrm{COCH}_{3}$ & & 61 & $-\mathrm{CH}_{2} \mathrm{Br}$ & 71 & $-\mathrm{COCH}_{3}$ \\
\hline & 43 & $-\mathrm{CH}_{2} \mathrm{Cl}$ & 53 & $-\mathrm{CH}=\mathrm{CH}_{2}$ & & 62 & $-\mathrm{CH}_{2} \mathrm{Cl}$ & 72 & $-\mathrm{CH}=\mathrm{CH}_{2}$ \\
\hline & 44 & $-\mathrm{NO}_{2}$ & 54 & $-\mathrm{CH}_{3}$ & & 63 & $-\mathrm{NO}_{2}$ & 73 & $-\mathrm{CH}_{3}$ \\
\hline & 45 & $-\mathrm{NH}_{2}$ & 55 & $-\mathrm{OCH}_{3}$ & & 64 & $-\mathrm{NH}_{2}$ & 74 & $-\mathrm{OCH}_{3}$ \\
\hline & 46 & $-\mathrm{Cl}$ & 56 & $-\mathrm{OCH}_{2} \mathrm{CH}_{3}$ & & 65 & $-\mathrm{Cl}$ & 75 & $-\mathrm{OCH}_{2} \mathrm{CH}_{3}$ \\
\hline & 47 & $-\mathrm{Br}$ & 57 & $-\mathrm{OH}$ & & 66 & $-\mathrm{Br}$ & 76 & $-\mathrm{OH}$ \\
\hline & 48 & -NHAc & 58 & $-\mathrm{COBr}$ & & 67 & -NHAc & 77 & $-\mathrm{COBr}$ \\
\hline & 49 & $-\mathrm{COCl}$ & 59 & $-\mathrm{CN}$ & & 68 & $-\mathrm{COCl}$ & 78 & $-\mathrm{CN}$ \\
\hline & 50 & $-\mathrm{COOH}$ & & & & 69 & $-\mathrm{COOH}$ & & \\
\hline Parent compound & \multicolumn{4}{|c|}{ Alkyl Group (R) } & Parent compound & \multicolumn{4}{|c|}{ Alkyl Group (R) } \\
\hline & 79 & $-\mathrm{CH}_{2} \mathrm{OH}$ & 89 & $-\mathrm{CHO}$ & & 98 & $-\mathrm{CH}_{2} \mathrm{OH}$ & 108 & $-\mathrm{CHO}$ \\
\hline
\end{tabular}




\begin{tabular}{|c|c|c|c|c|c|c|c|c|}
\hline & 80 & $-\mathrm{CH}_{2} \mathrm{Br}$ & 90 & $-\mathrm{COCH}_{3}$ & 99 & $-\mathrm{CH}_{2} \mathrm{Br}$ & 109 & $-\mathrm{COCH}_{3}$ \\
\hline & 81 & $-\mathrm{CH}_{2} \mathrm{Cl}$ & 91 & $-\mathrm{CH}=\mathrm{CH}_{2}$ & 100 & $-\mathrm{CH}_{2} \mathrm{Cl}$ & 110 & $-\mathrm{CH}=\mathrm{CH}_{2}$ \\
\hline & 82 & $-\mathrm{NO}_{2}$ & 92 & $-\mathrm{CH}_{3}$ & 101 & $-\mathrm{NO}_{2}$ & 111 & $-\mathrm{CH}_{3}$ \\
\hline & 83 & $-\mathrm{NH}_{2}$ & 93 & $-\mathrm{OCH}_{3}$ & 102 & $-\mathrm{NH}_{2}$ & 112 & $-\mathrm{OCH}_{3}$ \\
\hline & 84 & $-\mathrm{Cl}$ & 94 & $-\mathrm{OCH}_{2} \mathrm{CH}_{3}$ & 103 & $-\mathrm{Cl}$ & 113 & $-\mathrm{OCH}_{2} \mathrm{CH}_{3}$ \\
\hline & 85 & $-\mathrm{Br}$ & 95 & $-\mathrm{OH}$ & 104 & $-\mathrm{Br}$ & 114 & $-\mathrm{OH}$ \\
\hline & 86 & -NHAc & 96 & $-\mathrm{COBr}$ & 105 & -NHAc & 115 & $-\mathrm{COBr}$ \\
\hline & 87 & $-\mathrm{COCl}$ & 97 & $-\mathrm{CN}$ & 106 & $-\mathrm{COCl}$ & 116 & $-\mathrm{CN}$ \\
\hline & 88 & $-\mathrm{COOH}$ & & & 107 & $-\mathrm{COOH}$ & & \\
\hline
\end{tabular}

\subsection{Preparation of protein and noscapine}

Protease of SARS-CoV-2 (PDB-6LU7) is prepared before docking within UCSF Chimera 1.11.2.(Pettersen et al., 2004) Initially pre-existed ligand and water molecule were removed, hydrogen was added, incomplete residues were repaired and finally charge were assigned by applying AMBER.ff14SB force field via the dock prep module. This prepared pdb 6LU7 is used for molecular docking and virtual screening. Noscapines were also geometrically optimized to remove the steric clash present within the molecule. Steric energy was minimized by Chem3D applying molecular mechanics as a force field and minimum root mean square gradient was set to 0.01.("Chem3D http://www.cambridesoft.com,") These optimized noscapines were used to inhibition of protease of SARS-CoV-2.

\subsection{Molecular Docking}

Computational docking gives us precise and accurate possible interaction of a drug with the protein. It also uses algorithms to provide some physical parameter to quantify the interaction.(Tomlinson et al., 2009; V. K. S. Vishvakarma, N.; Reetu; Kumari, K.; Patel R.; Singh, P., 2019) All 116 noscapines were docked against the protease of SARS-CoV-2 for the allosteric inhibition. Molecular docking was performed by the iGEMDOCK v2.1.(Yang $\&$ Chen, 2004) Here no binding pocket was defined for docking. iGEMDOCK was set into the drug screening mode. In this mode number of solution for each drug is searched thrice, each solution has seventy generations and each generation have 200 population. The total binding energy of noscapine with the amino acid of the active cavity is measured in terms of hydrogen bonding energy, van der Waals energy and electrostatic interaction energy.(Chakravarty, Singh, \& Kumari, 2016; Kumar, Kumari, Jayaraj, Kumar, Singh et al., 
2020; Kumar, Kumari, Jayaraj, \& Singh, 2020; Kumar, Singh, Chandra, Kumari, \& Kumar, 2017; Singh, Kumari, Awasthi, \& Chandra, 2016; Singh, Kumari, \& Chandra, 2016a, 2016b; Singh et al., 2017; V. K. Vishvakarma et al., 2015; V. K. Vishvakarma, Patel, Kumari, \& Singh, 2017; V. K. Vishvakarma, Shukla et al., 2019; V. K. Vishvakarma, Singh et al., 2019; V. K. Vishvakarma, Singh, Kumari, \& Chandra, 2017)

\subsection{Post-Docking analysis and modeling}

Post dock modeling provides a pictorial view to see the interaction between the noscapines and COVID-19 protease. An atomic level interaction with its distance is analyzed by Discovery Studio Visualizer V-2017.2 of BIOVIA.(BIOVIA, 2017) Only classical hydrogen bonds with distance is showed in 3D view while other possible interactions like non-classical hydrogen bond, hydrophobic interaction and electrostatic energy along with hydrogen bonds were also shown $2 \mathrm{D}$ view.

\subsection{ADMET properties}

Physicochemical descriptors act as a marker to define the probable properties of the molecule to be a drug.(Celik, Albayrak, Akyuz, \& Ozel, 2019) These descriptors are partition coefficient $(\log \mathrm{P})$, heavy atoms, Molecular weight (MW), aromatic heavy atoms, no. of rotatable bonds, H-bond donors, H-bond acceptors, topological polar surface area (TPSA) solubility $(\log S)$, distribution coefficient $\left(\log \mathrm{D}_{7.4}\right)$, etc. Based on these physicochemical descriptors absorption, distribution, metabolism, excretion and toxicity (ADMET) properties of top-six noscapines were calculated using a web server (http://admet.scbdd.com/calcpre/index/).

\section{Result}

\subsection{Molecular docking result}

Molecular interaction provides by molecular docking indicates the probable anchoring of the noscapine into the active binding cavity of protease of SARS-CoV-2. These interactions are van der Waal, hydrogen bonding and electrostatic interaction.(Kumar et al., 2019a; V. K. S. Vishvakarma, N.; Reetu; Kumari, K.; Patel R.; Singh, P., 2019) Sum of the energy-related to corresponding interaction will to leads to the total binding energy and act as a marker for particular noscapines regarding their potential against the protease of SARS-CoV-2.(Azam \& Jupudi, 2019) Based on the total binding energy, noscapines were screened and arranged by their potential as given in Table 2. 
Table 2 Docking result of the 116 noscapines against COVID-19 protease

\begin{tabular}{|c|c|c|c|c|c|}
\hline Noscapines & T Energy & VDW & H Bond & Elec & $\begin{array}{l}\text { Aver Con } \\
\text { Pair }\end{array}$ \\
\hline Nos 107 & -137.66 & -111.04 & -25.7247 & -0.89556 & 19.0952 \\
\hline Nos37 & -136.265 & -121.787 & -14.4778 & 0 & 22.0513 \\
\hline Nos 86 & -129.945 & -109.02 & -20.9253 & 0 & 22.1136 \\
\hline Nos88 & -128.997 & -96.4177 & -29.3132 & -3.26615 & 20.9762 \\
\hline Nos 83 & -127.897 & -91.1272 & -36.7701 & 0 & 19.9737 \\
\hline Nos41 & -127.879 & -106.593 & -21.2863 & 0 & 20.8947 \\
\hline Nos 104 & -127.121 & -113.75 & -13.3709 & 0 & 21.1579 \\
\hline Nos 87 & -126.804 & -112.83 & -13.974 & 0 & 19.9048 \\
\hline Nos42 & -126.494 & -109.404 & -17.09 & 0 & 20.3158 \\
\hline Nos 106 & -126.334 & -108.793 & -17.5408 & 0 & 18.5714 \\
\hline Nos 82 & -124.954 & -104.006 & -19.6615 & -1.28617 & 18.381 \\
\hline Nos44 & -124.880 & -95.5684 & -29.3113 & 0 & 17.9744 \\
\hline Nos68 & -124.163 & -120.918 & -3.2454 & 0 & 18.5897 \\
\hline Nos40 & -123.782 & -91.1748 & -32.6074 & 0 & 20.6316 \\
\hline Nos92 & -123.268 & -108.13 & -15.1381 & 0 & 20.7105 \\
\hline Nos59 & -123.259 & -109.927 & -13.3316 & 0 & 21.2895 \\
\hline Nos 110 & -122.863 & -100.904 & -21.9597 & 0 & 22.625 \\
\hline Nos26 & -122.853 & -106.591 & -16.2628 & 0 & 21.8919 \\
\hline Nos55 & -122.626 & -113.244 & -9.38157 & 0 & 20.7895 \\
\hline Nos91 & -122.400 & -101.507 & -20.8927 & 0 & 17.3 \\
\hline Nos 102 & -122.285 & -80.2054 & -42.08 & 0 & 20.5789 \\
\hline Nos45 & -121.754 & & -8.80392 & 0 & 22.1351 \\
\hline Nos80 & -121.602 & -107.146 & -14.4559 & 0 & 17.925 \\
\hline Nos77 & -121.484 & -95.3802 & -26.1041 & 0 & 18.8974 \\
\hline Nos60 & -121.465 & -104.274 & -17.1913 & 0 & 20.0789 \\
\hline Nos67 & -120.756 & -105.539 & -15.2167 & 0 & 19.9 \\
\hline Nos78 & -120.731 & -107.819 & -12.9122 & 0 & 21.4211 \\
\hline Nos75 & -120.661 & -107.068 & -13.5932 & 0 & 20.5385 \\
\hline Nos 116 & -120.58 & -101.914 & -18.6668 & 0 & 16.65 \\
\hline Nos32 & -120.347 & -97.0016 & -23.3455 & 0 & 21.0263 \\
\hline Nos 109 & -120.277 & -107.624 & -12.6525 & 0 & 19.0238 \\
\hline Nos79 & -119.887 & -106.329 & -13.5583 & 0 & 19.975 \\
\hline Nos98 & -119.822 & -90.6873 & -29.1352 & 0 & 18.15 \\
\hline Nos69 & -119.71 & -116.31 & -4.65159 & 1.2517 & 19.4103 \\
\hline Nos64 & -119.545 & -107.698 & -11.8468 & 0 & 21.0811 \\
\hline Nos58 & -118.865 & -110.5 & -8.36495 & 0 & 20.9487 \\
\hline Nos 101 & -118.51 & -94.148 & -25.3406 & 0.978742 & 16.5476 \\
\hline Nos74 & -118.458 & -111.215 & -7.24267 & 0 & 19.8158 \\
\hline Nos56 & -118.269 & -101.657 & -16.612 & 0 & 19.1026 \\
\hline Nos7 & -118.166 & -108.132 & -10.0335 & 0 & 23 \\
\hline Nos95 & -118.158 & -110.892 & -7.26576 & 0 & 18.6053 \\
\hline
\end{tabular}




\begin{tabular}{|c|c|c|c|c|c|}
\hline Nos115 & -118.108 & -113.34 & -4.76835 & 0 & 18.8571 \\
\hline Nos 21 & -118.098 & -105.971 & -12.1269 & 0 & 21.5833 \\
\hline Nos46 & -117.985 & -95.7005 & -22.2841 & 0 & 21.3784 \\
\hline Nos38 & -117.953 & -100.904 & -17.049 & 0 & 19.9459 \\
\hline Nos23 & -117.827 & -98.9312 & -18.8961 & 0 & 19.6316 \\
\hline Nos90 & -117.819 & -111.189 & -6.62963 & 0 & 20.1429 \\
\hline Nos57 & -117.772 & -90.3229 & -27.4495 & 0 & 27.5946 \\
\hline Nos94 & -117.7 & -105.986 & -11.7145 & 0 & 17.9286 \\
\hline Nos43 & -117.522 & -104.775 & -12.747 & 0 & 19.6842 \\
\hline Nos35 & -117.369 & -113.869 & -3.5 & 0 & 21.2703 \\
\hline Nos113 & -117.324 & -95.5787 & -21.7458 & 0 & 18.5952 \\
\hline Nos 108 & -117.247 & -106.725 & -10.5224 & 0 & 18.125 \\
\hline Nos4 & -116.78 & -109.78 & -7 & 0 & 23.5313 \\
\hline Nos96 & -116.654 & -90.9813 & -25.673 & 0 & 16.5952 \\
\hline Nos112 & -116.576 & -100.4 & -16.1763 & 0 & 20.6 \\
\hline Nos51 & -115.844 & -107.097 & -8.74648 & 0 & 19.4474 \\
\hline Nos81 & -115.644 & -98.1043 & -17.5396 & 0 & 18.025 \\
\hline Nos 85 & -115.483 & -95.9456 & -19.5377 & 0 & 19.0526 \\
\hline Nos29 & -115.328 & -109.175 & -6.15279 & 0 & 18.775 \\
\hline Nos63 & -115.238 & -85.3582 & -30.4642 & 0.584563 & 15.4359 \\
\hline Nos93 & -114.961 & -97.1946 & -17.7666 & 0 & 19.175 \\
\hline Nos5 & -114.349 & -99.8087 & -13.9128 & -0.62741 & 23.8485 \\
\hline Nos84 & -113.949 & -110.224 & -3.72575 & 0 & 17.9474 \\
\hline Nos31 & -113.637 & -86.6982 & -25.9131 & -1.0255 & 21.2564 \\
\hline Nos114 & -113.563 & -98.3684 & -15.1946 & 0 & 19.7895 \\
\hline Nos 25 & -113.563 & -107.852 & -4.99133 & -0.72034 & 19.4103 \\
\hline Nos6 & -113.397 & -100.074 & -13.3234 & 0 & 22.9032 \\
\hline Nos 105 & -112.871 & -103.084 & -9.78685 & 0 & 16.5455 \\
\hline Nos33 & -112.674 & -101.118 & -11.5564 & 0 & 19.2821 \\
\hline Nos28 & -112.446 & -110.164 & -2.2829 & 0 & 19.0811 \\
\hline Nos13 & -112.386 & -85.6749 & -26.7111 & 0 & 18.9697 \\
\hline Nos76 & -112.325 & -100.604 & -11.7218 & 0 & 21.2432 \\
\hline Nos39 & -111.241 & -96.6882 & -14.553 & 0 & 17.5128 \\
\hline Nos47 & -111.23 & -110.069 & -1.16113 & 0 & 20.2703 \\
\hline Nos70 & -111.024 & -96.3665 & -14.6579 & 0 & 18.1053 \\
\hline Nos49 & -110.968 & -100.662 & -10.3062 & 0 & 21.4359 \\
\hline Nos36 & -110.739 & -101.918 & -8.82085 & 0 & 20.8947 \\
\hline Nos30 & -110.68 & -90.8393 & -19.841 & 0 & 17.4103 \\
\hline Nos11 & -110.43 & -78.665 & -29.0969 & -2.66823 & 18.6667 \\
\hline Nos73 & -110.368 & -99.5901 & -10.778 & 0 & 21.5676 \\
\hline Nos111 & -109.755 & -105.481 & -4.27339 & 0 & 19.1579 \\
\hline Nos71 & -109.628 & -82.9617 & -26.6662 & 0 & 18.4359 \\
\hline Nos97 & -109.168 & -98.2182 & -10.9501 & 0 & 15.875 \\
\hline Nos99 & -108.684 & -99.8294 & -8.85468 & 0 & 19.825 \\
\hline
\end{tabular}




\begin{tabular}{|c|c|c|c|c|c|}
\hline Nos 19 & -108.56 & -88.6824 & -19.878 & 0 & 21.9697 \\
\hline Nos61 & -108.153 & -106.203 & -1.95035 & 0 & 19.6842 \\
\hline Nos89 & -108.099 & -90.6922 & -17.4065 & 0 & 18.3 \\
\hline Nos66 & -107.958 & -107.958 & 0 & 0 & 18.0811 \\
\hline Nos22 & -107.872 & -80.5735 & -27.2987 & 0 & 14.7632 \\
\hline Nos 100 & -107.743 & -104.936 & -2.80724 & 0 & 18.075 \\
\hline Nos12 & -107.584 & -85.8111 & -21.7733 & 0 & 18.7188 \\
\hline Nos62 & -107.35 & -98.7481 & -8.60176 & 0 & 16.1579 \\
\hline Nos9 & -106.953 & -101.063 & -5.8903 & 0 & 22.1176 \\
\hline Nos24 & -106.913 & -101.177 & -5.73577 & 0 & 19 \\
\hline Nos10 & -106.885 & -82.3387 & -24.5466 & 0 & 18.9091 \\
\hline Nos8 & -106.718 & -79.8898 & -26.8278 & 0 & 20.5484 \\
\hline Nos20 & -106.653 & -76.9796 & -29.6732 & 0 & 18.4063 \\
\hline Nos2 & -106.577 & -98.2542 & -8.32291 & 0 & 22.6563 \\
\hline Nos52 & -106.181 & -102.681 & -3.5 & 0 & 19.5385 \\
\hline Nos34 & -105.721 & -86.6385 & -19.0829 & 0 & 16.7895 \\
\hline Nos53 & -105.347 & -100.132 & -5.21481 & 0 & 18.2368 \\
\hline Nos 15 & -104.812 & -100.065 & -4.747 & 0 & 22.129 \\
\hline Nos 16 & -104.795 & -85.9283 & -18.8662 & 0 & 19.3438 \\
\hline Nos 14 & -104.286 & -94.1771 & -10.1084 & 0 & 21.4688 \\
\hline Nos 18 & -104.2 & -71.2975 & -32.9022 & 0 & 17.4194 \\
\hline Nos 27 & -103.847 & -101.347 & -2.5 & 0 & 19.027 \\
\hline Nos17 & -103.565 & -89.1215 & -14.444 & 0 & 21.2727 \\
\hline Nos54 & -101.967 & -99.5552 & -2.41173 & 0 & 16.973 \\
\hline Nos48 & -101.53 & -95.7947 & -5.73481 & 0 & 15.375 \\
\hline Nos103 & -100.611 & -82.2533 & -18.3581 & 0 & 16.8421 \\
\hline Nos50 & -99.0625 & -92.0625 & -7 & 0 & 18.3077 \\
\hline Nos3 & -98.8533 & -80.2924 & -18.5609 & 0 & 18.0625 \\
\hline Nos65 & -98.0369 & -88.4149 & -9.62195 & 0 & 19.7568 \\
\hline Nos72 & -96.3163 & -75.6863 & -20.63 & 0 & 19.0789 \\
\hline Nos 1 & -94.3241 & -81.699 & -12.625 & 0 & 18.4333 \\
\hline
\end{tabular}

(VDW = van der Waals interaction energy, H-bond = hydrogen bonding energy; Elec = Electrostatic ineraction energy; Aver Con Pair = Average confirmation pair)

Further, 14 known compounds with different antiviral potential were taken from the database and our results were compared with the reported antiviral agents. The reported molecules are N3 (co-crystallized inhibitor of 6LU7), Camostat (SARS-CoV inhibitor), Remdesivir (antiviral medicine for Ebola), Baricitinib (inhibitor of Janus kinases), Favipiravir (Influenza antiviral), Galidesivir (Ebola antiviral), Darunavir-2 (HIV/AIDS antiviral), Thalidomide (anticancerous), Cobicistat (HIV/AIDS antiviral), Ruxolitinib (antineoplastic), Fingolimod (sphingosine 1-phosphate receptor modulators), Hydroxychloroquine (antimalarial), 
Chloroquine (antimalarial), Arbidol (influenza antiviral). Docking results of 14 known antiviral drugs are given in Table 3.

Table 3 Docking result 14 well known antiviral drugs

\begin{tabular}{|l|l|l|l|l|}
\hline Compound name & T. Energy & VDW & H Bond & Elec \\
\hline N3 & -116.132 & -104.716 & -11.4159 & 0 \\
\hline Camostat & -114.554 & -94.6993 & -17.4391 & -2.41559 \\
\hline Remdesivir & -105.955 & -82.4292 & -23.5262 & 0 \\
\hline Baricitinib & -94.5708 & -62.9297 & -31.641 & 0 \\
\hline Favipiravir & -93.8858 & -57.7481 & -36.1377 & 0 \\
\hline Galidesivir & -91.6304 & -59.05 & -32.5804 & 0 \\
\hline Darunavir-2 & -91.3952 & -73.1994 & -18.1957 & 0 \\
\hline Thalidomide & -88.7425 & -69.6454 & -19.097 & 0 \\
\hline Cobicistat & -83.7343 & -74.1677 & -9.56651 & 0 \\
\hline Ruxolitinib & -82.5082 & -71.6024 & -10.9059 & 0 \\
\hline Fingolimod & -75.6867 & -60.3308 & -15.3559 & 0 \\
\hline Hydroxychloroquine & -74.8428 & -66.1241 & -8.71866 & 0 \\
\hline Chloroquine & -73.894 & -65.431 & -8.463 & 0 \\
\hline Arbidol & -69.6036 & -63.6572 & -5.9464 & 0 \\
\hline
\end{tabular}

After comparison of the docking result of noscapines with the 14 well known antiviral drugs, it is found that most of the noscapines have the least binding energy that the top of the well antiviral drug (N3). From there, the top six noscapines were taken for ADMET studies. These noscapines are nos $107, \operatorname{nos} 37, \operatorname{nos} 86, \operatorname{nos} 88, \operatorname{nos} 83$ and nos 41 respectively and given in Table 4.

Table 4 Top six noscapines against the protease of SARS-CoV-2

\begin{tabular}{|l|r|r|r|r|r|}
\hline Ligand & \multicolumn{1}{l|}{$\begin{array}{l}\text { Total } \\
\text { Energy }\end{array}$} & \multicolumn{1}{l|}{ VDW } & H Bond & \multicolumn{1}{l|}{ Elec } & \multicolumn{1}{l|}{$\begin{array}{l}\text { Aver Con } \\
\text { Pair }\end{array}$} \\
\hline Nos107 & -137.66 & -111.04 & -25.7247 & -0.8955 & 19.0952 \\
\hline Nos37 & -136.265 & -121.787 & -14.4778 & 0 & 22.0513 \\
\hline Nos86 & -129.945 & -109.02 & -20.9253 & 0 & 22.1136 \\
\hline Nos88 & -128.997 & -96.4177 & -29.3132 & -3.2661 & 20.9762 \\
\hline Nos83 & -127.897 & -91.1272 & -36.7701 & 0 & 19.9737 \\
\hline Nos41 & -127.879 & -106.593 & -21.2863 & 0 & 20.8947 \\
\hline
\end{tabular}

Total binding energy of top six noscapines are $-137.66,-136.265,129.945,128.997,127.897$ and $-127.879 \mathrm{KJ} / \mathrm{mol}$ respectively. Lowest van der Waal contribution is found for nos37 among the top six, the lowest hydrogen bonding contribution is found for Nos83 and the lowest electrostatic contribution is found for nos88 among the top six. Based on the total binding energy of docking of top-six noscapines were analyzed by the number of interaction and distance between the atoms of noscapines and COVID-19 protease. Classical hydrogen 
bonding, non-classical hydrogen bonding, electrostatic and hydrophobic interaction of top-six noscapines were given in Figure 1.
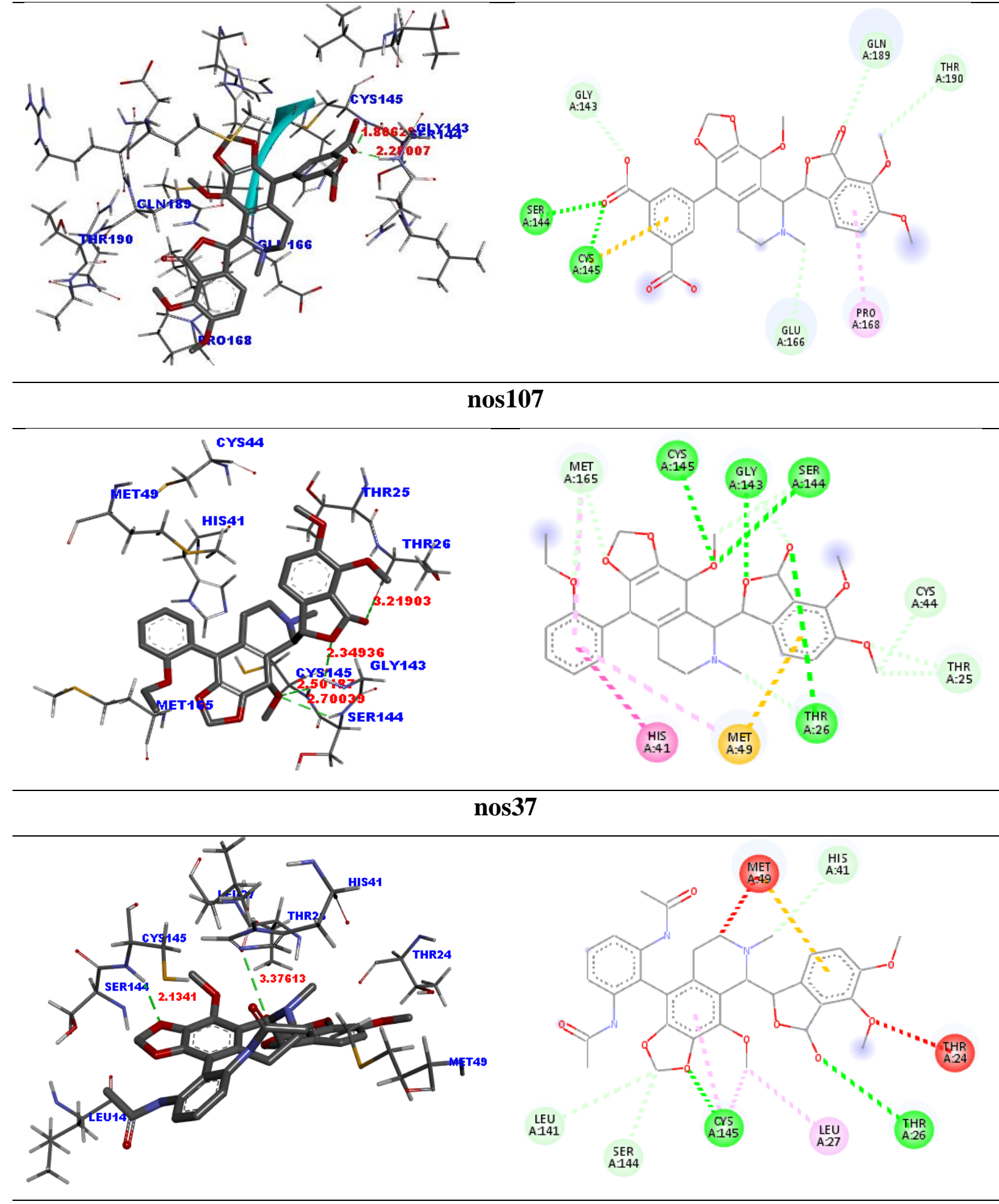

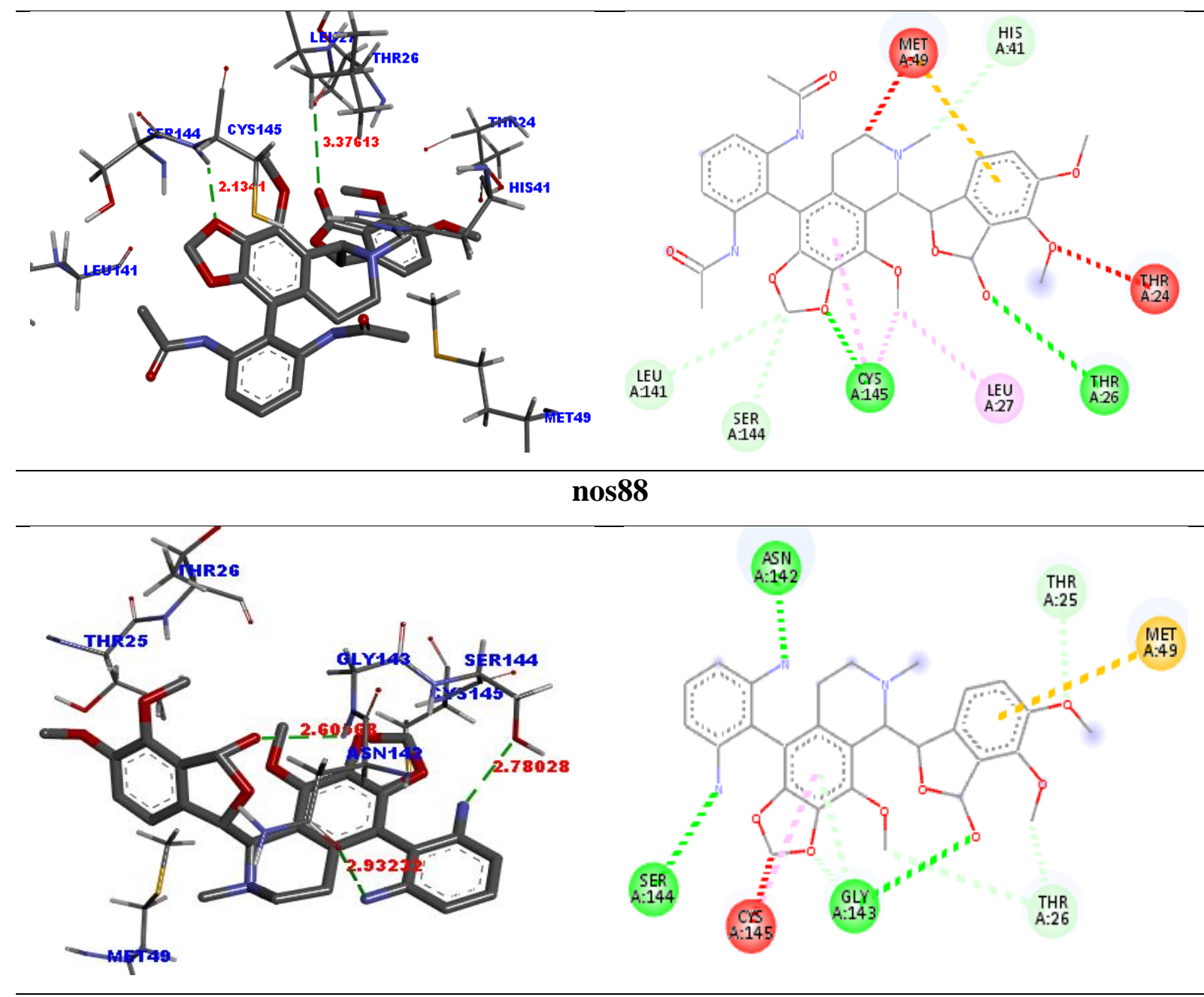

nos83

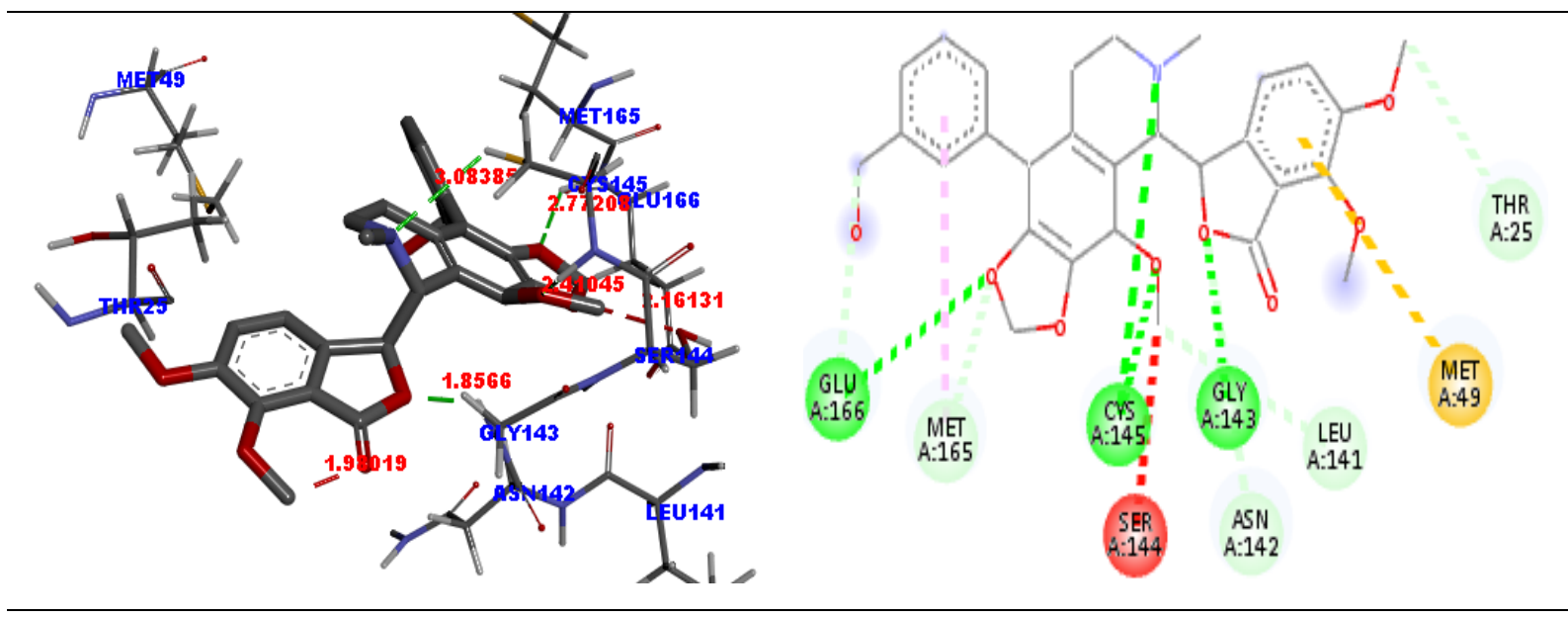

nos41

Interactions

Unfavorable Bump

Conventional Hydrogen Bond

Carbon Hydrogen Bond

Figure 2 Details of interactions formed

between the atoms of nos $107, \operatorname{nos} 37, \operatorname{nos} 86$,

nos88, nos83 and nos41 with COVID-19 protease 
A detailed interaction along with the distance of interaction of top-six noscapines with the various amino acids of the active cavity of protease of SARS-CoV-2 is given in Table 5.

Table 5 Interaction of the top six noscapines with amino-acids of the protease of SARS-CoV-

\begin{tabular}{|c|c|c|c|c|c|c|}
\hline \multirow[t]{3}{*}{ Nos } & \multicolumn{4}{|c|}{ H-Bond } & \multicolumn{2}{|c|}{ Hydrophobic } \\
\hline & \multicolumn{2}{|c|}{ Classical } & \multicolumn{2}{|c|}{ Non-classical } & & \\
\hline & $\begin{array}{l}\text { Amino } \\
\text { Acid }\end{array}$ & $\begin{array}{l}\text { Distance } \\
\text { (̊) }\end{array}$ & $\begin{array}{l}\text { Amino } \\
\text { Acid }\end{array}$ & $\begin{array}{l}\text { Distance } \\
\text { (̊) }\end{array}$ & $\begin{array}{l}\text { Amino } \\
\text { Acid }\end{array}$ & $\begin{array}{l}\text { Distance } \\
\text { (̊) }\end{array}$ \\
\hline \multirow{4}{*}{$\begin{array}{l}\text { Nos } \\
107\end{array}$} & SER 144 & 2.28 & GLY 143 & 2.43 & PRO 168 & 5.06 \\
\hline & CYS 145 & 1.80 & GLU 166 & 3.03 & & \\
\hline & & & GLN 189 & 2.62 & & \\
\hline & & & THR 190 & 3.26 & & \\
\hline \multirow{6}{*}{$\begin{array}{l}\text { Nos } \\
37\end{array}$} & THR 26 & 3.21 & MET 165 & $2.68 ; 2.88$ & MET 49 & 5.29 \\
\hline & CYS 145 & 2.50 & SER 144 & 2.80 & HIS 41 & 4.39 \\
\hline & GLY 143 & 2.34 & GLY 143 & $2.67 ; 2.93$ & MET 165 & 5.49 \\
\hline & SER 144 & 2.70 & THR 26 & 3.04 & & \\
\hline & & & CYS 44 & 3.62 & & \\
\hline & & & THR 25 & $2.43 ; 2.60$ & & \\
\hline \multirow{3}{*}{$\begin{array}{l}\text { Nos } \\
86\end{array}$} & CYS 145 & 2.13 & LEU 141 & 3.06 & CYS 145 & $3.85 ; 3.31$ \\
\hline & THR 26 & 3.37 & SER 144 & 3.01 & LEU 27 & 4.23 \\
\hline & & & HIS 41 & 3.31 & & \\
\hline \multirow{3}{*}{$\begin{array}{l}\text { Nos } \\
88\end{array}$} & CYS 145 & 2.13 & LEU 141 & 3.01 & LEU 27 & 4.23 \\
\hline & THR 26 & 3.37 & SER 144 & 3.06 & CYS 145 & $3.85 ; 3.31$ \\
\hline & & & HIS 41 & 2.24 & & \\
\hline \multirow{3}{*}{$\begin{array}{l}\text { Nos } \\
83\end{array}$} & ASN 142 & 2.93 & THR 25 & 2.70 & CYS 145 & 4.51 \\
\hline & SER 144 & 2.78 & ARG 26 & $3.29 ; 3.76$ & & \\
\hline & GLY 143 & 2.60 & GLY 143 & $2.96 ; 2.87$ & & \\
\hline \multirow{6}{*}{$\begin{array}{l}\text { Nos } \\
41\end{array}$} & GLU 166 & 2.77 & MET 165 & 2.25 & MET 165 & 4.81 \\
\hline & CYS 145 & $2.41 ; 3.08$ & GLU 166 & 3.59 & & \\
\hline & GLY 143 & 1.85 & LEU 141 & 2.69 & & \\
\hline & & & SER 144 & 2.16 & & \\
\hline & & & ASN 142 & 2.99 & & \\
\hline & & & THR 25 & 3.29 & & \\
\hline
\end{tabular}

When noscapine binds with the atoms of active amino acids with the help of hydrogen bonding, electrostatic interaction, hydrophobic interaction, etc. then, different amino acid have their corresponding binding energy. A detailed graphical analysis of amino acid and its corresponding stabilization for the top six noscapines is given in Figure 2. A highly interesting to note that HIS-41, GLY-143, CYS-145, GLU-166, ASN-142, HIS-164, MET165, GLU-166, GLN-189, MET-49 and THR-199 are the common amino acid residues of the active cavity. The same active cavity is targeted by all top six noscapines. 


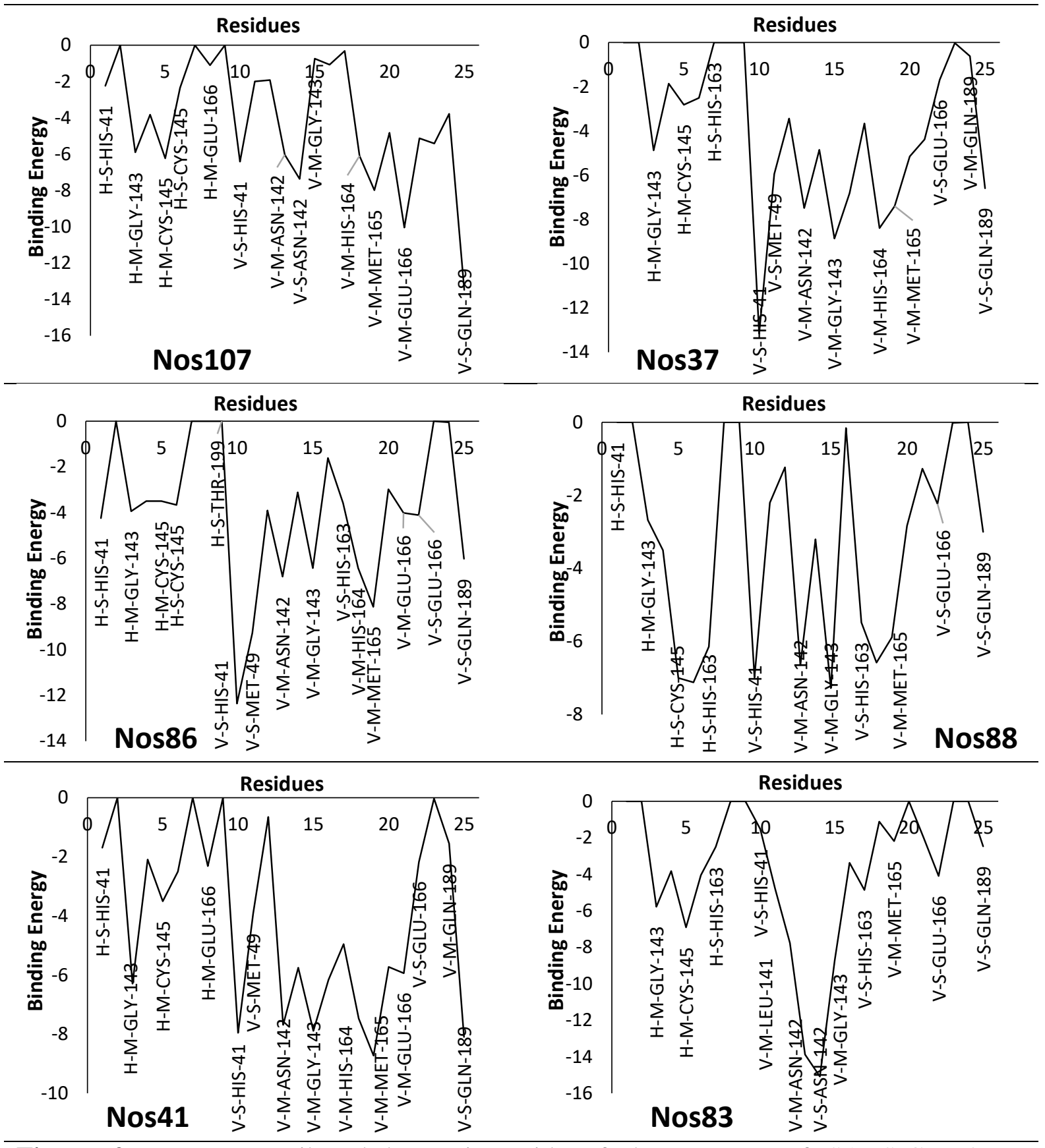

Figure 2 Energy contributed by amino-acids of the protease of SARS-CoV-2 on interaction with the top-six noscapines

\subsection{ADMET Result}

ADMET properties of top-six noscapines are analyzed based on the physicochemical descriptors.(Ferreira \& Andricopulo, 2019) These physicochemical descriptors are molecular weight (M. W.), hydrogen bond acceptor atoms (HB Accep), hydrogen bond donor atoms (HB Donor), topological polar surface area (TPSA), solubility ( $\log S)$, lipophilicity (Log P) and distribution coefficient $\left(\log \mathrm{D}_{7.4}\right)$. The values of these descriptors are given in Table 6. Based on these descriptors ADMET properties of the top six noscapines were determined. 
Table $6 \operatorname{LogS}, \log \mathrm{D} 7.4$ and $\log \mathrm{P}$ of the top six compounds

\begin{tabular}{|l|l|l|l|l|l|l|}
\hline Property & \multicolumn{1}{|c|}{$\mathbf{1 0 7}$} & \multicolumn{1}{c|}{$\mathbf{3 7}$} & \multicolumn{1}{c|}{$\mathbf{8 6}$} & \multicolumn{1}{c|}{$\mathbf{8 8}$} & \multicolumn{1}{c|}{$\mathbf{8 3}$} & \multicolumn{1}{c|}{$\mathbf{4 1}$} \\
\hline M. W. & 577.542 & 533.577 & 603.628 & 577.542 & 519.554 & 519.55 \\
\hline HB Accep & 10 & 9 & 10 & 10 & 10 & 9 \\
\hline HB Donor & 2 & 0 & 2 & 2 & 2 & 1 \\
\hline TPSA & 150.29 & 84.92 & 133.89 & 150.29 & 127.73 & 95.92 \\
\hline Log S & -4.164 & -5.323 & -4.862 & -4.157 & -4.397 & -4.431 \\
\hline LogD7.4 & 1.068 & 1.645 & 1.569 & 1.043 & 1.157 & 1.171 \\
\hline LogP & 3.945 & 4.948 & 4.466 & 3.945 & 3.713 & 4.041 \\
\hline
\end{tabular}

\subsubsection{Absorption properties of the top six noscapines}

Absorption in term of human intestinal absorption (HIA), bioavailability (F20\% \& F30\%), Caco-2 permeability and permeability glycoprotein (P-gp) for inhibitor and substrate was analyzed for top six noscapines as given in Table 7. The rate of reflux across is measured in terms of Caco-2 cells permeability.(Lule et al., 2020) Except nos37, all noscapines have optimum permeability for Caco-2 cells. Glycoproteins are the part of the cell membrane and its permeability depends on the lipophilicity value.(Feldmann et al., 2014) It's measured in terms of probability percent and the highest Pgp-inhibitor value is found for nos37 and Pgpsubstrate for Nos41. Most of the drug is absorbed by the intestine known as human intestinal absorption (HIA).(Feinberg, Pande, \& Cheng, 2019) The highest intestinal absorption was found for nos37. Bioavailability in terms of F20\% and F30\% were also measured in terms of probability.(Di, Kerns, \& Carter, 2009) Highest F30\% probability is found for nos83 while the highest $\mathrm{F} 30 \%$ probability is found for nos 88 .

Table 7 Absorption properties of the top six noscapines

\begin{tabular}{|l|l|l|l|l|l|l|}
\hline Property & \multicolumn{1}{|c|}{$\mathbf{1 0 7}$} & \multicolumn{1}{|c|}{$\mathbf{3 7}$} & $\mathbf{8 6}$ & $\mathbf{8 8}$ & $\mathbf{8 3}$ & \multicolumn{1}{|c|}{} \\
\hline Caco-2 Permeability & -5.359 & -5.022 & -5.181 & -5.354 & -5.416 & -5.193 \\
\hline Pgp-inhibitor & 0.799 & 0.91 & 0.865 & 0.716 & 0.693 & 0.900 \\
\hline Pgp-substrate & 0.222 & 0.257 & 0.241 & 0.194 & 0.391 & 0.259 \\
\hline HIA & 0.479 & 0.605 & 0.483 & 0.479 & 0.495 & 0.542 \\
\hline F (20\% Bioavailability) & 0.45 & 0.493 & 0.442 & 0.45 & 0.514 & 0.497 \\
\hline F (30\% Bioavailability) & 0.567 & 0.601 & 0.558 & 0.629 & 0.62 & 0.483 \\
\hline
\end{tabular}

\subsubsection{Distribution properties of top-six noscapines}

Distribution of drugs within the patient's body is most important and also known as pharmacokinetics.(Di et al., 2009) It's measured in terms of blood-brain barrier penetration (BBB), volume distribution (VD) and plasma protein binding (PPB). Values corresponding to the distribution parameters is given in Table 8.

Table 8 Distribution properties of top six noscapines 


\begin{tabular}{|c|c|c|c|c|c|c|}
\hline Pro & 107 & 37 & 86 & 88 & 83 & 41 \\
\hline PPB & 86.027 & 80.052 & 79.961 & 83.558 & 78.389 & 83.144 \\
\hline & 0.0 & 0.4 & 0.207 & -0.107 & 0.303 & 0.556 \\
\hline BBB & 0.619 & 0.939 & 0.738 & 0.64 & 0.946 & 0.923 \\
\hline
\end{tabular}

Plasma protein act as a carrier for the noscapine within the blood. When a drug binds with plasma protein at also act as a reservoir hence responsible for the release of the drug. So, less the plasma protein binding more will be distributed.(Ma et al., 2008) Lowest PPB value is found for nos83. The volume of drugs in the bloodstream is very important for the further release of the drug into the bloodstream from plasma protein. $\mathrm{VD}<0.07 \mathrm{~L} / \mathrm{kg}$ corresponds to bind with plasma protein, VD 0.07-0.7 L/kg corresponds to evenly distribution and VD > 0.7 $\mathrm{L} / \mathrm{kg}$ corresponds to distribution towards tissue components.(Li, Yan, Wang, \& Yu, 2019) Highest value of VD is found for nos41. Drugs that can cross BBB may act on the central nervous system (CNS). Values of BBB is categorized in two categories viz., BBB > 0.1 is $\mathrm{BBB}+$ and BB ratio <0.1 is BBB-.(Beard, Gaboriau, Gee, \& Tate, 2019) All top six noscapines shows $\mathrm{BBB}+$ nature.

\subsubsection{Metabolism properties of top-six noscapines}

Break down of drugs into the body is known as metabolism. Most of the break down is occurring in the liver. Redox enzymes are responsible for the breakdown and most common are cytochrome P450.(Xu \& Desta, 2013) After the metabolism of drug it breaks into pharmacologically active and inactive parts. Cytochrome P450 has many isozymes in which CYP1A2, CYP3A4, CYP2C9, CYP2C19 and CYP2D6 plays an important role.(Wu et al., 2019; Xu \& Desta, 2013) For top-six noscapines the probability values of these cytochromes in terms of the substrate (sub) and inhibitors (inh) were analyzed as in Table 9. The probability value of P450 CYP3A4 substrate was found highest for all top six noscapines.

Table 9 Metabolism properties of top six noscapines

\begin{tabular}{|l|l|l|l|l|l|l|}
\hline Property & \multicolumn{107}{|c|}{} & \multicolumn{1}{|c|}{$\mathbf{8 7}$} & $\mathbf{8 6}$ & $\mathbf{8 8}$ & $\mathbf{8 3}$ & $\mathbf{4 1}$ \\
\hline P450 CYP1A2 inh & 0.038 & 0.13 & 0.068 & 0.027 & 0.065 & 0.088 \\
\hline P450 CYP1A2 Sub & 0.458 & 0.531 & 0.547 & 0.515 & 0.443 & 0.472 \\
\hline P450 CYP3A4 inh & 0.379 & 0.757 & 0.537 & 0.338 & 0.689 & 0.645 \\
\hline P450 CYP3A4 sub & 0.69 & 0.779 & 0.681 & 0.667 & 0.661 & 0.686 \\
\hline P450 CYP2C9 inh & 0.546 & 0.731 & 0.491 & 0.47 & 0.527 & 0.572 \\
\hline P450 CYP2C9 sub & 0.376 & 0.379 & 0.354 & 0.352 & 0.27 & 0.359 \\
\hline P450 CYP2C19 inh & 0.258 & 0.734 & 0.506 & 0.199 & 0.598 & 0.61 \\
\hline P450 CYP2C19 sub & 0.534 & 0.577 & 0.47 & 0.466 & 0.455 & 0.532 \\
\hline P450 CYP2D6 inh & 0.53 & 0.539 & 0.498 & 0.496 & 0.543 & 0.541 \\
\hline P450 CYP2D6 sub & 0.525 & 0.568 & 0.498 & 0.474 & 0.533 & 0.541 \\
\hline
\end{tabular}




\subsubsection{Excretion properties of top-six noscapines}

Elimination of drug metabolites from the patient is most important to produce the least toxic effect. Excretion has several routes but through kidney and liver is best. The drugs which remain unchanged are mostly eliminated through the renal duct.(Daina, Michielin, \& Zoete, 2017; Guan et al., 2019) Mainly water-soluble drugs are excreted via urine. High lipophilic drugs mainly excreted through kidney.(Lipinski, 2000) Excretion properties of top-six noscapines were analyzed in terms of half-life $\left(\mathrm{t}_{1 / 2}\right)$ and clearance rate (CL) and values are given in Table 10.

Table 10 Excretion properties of top six noscapines

\begin{tabular}{|l|c|c|c|c|c|c|}
\hline Property & $\mathbf{1 0 7}$ & $\mathbf{3 7}$ & $\mathbf{8 6}$ & $\mathbf{8 8}$ & $\mathbf{8 3}$ & $\mathbf{4 1}$ \\
\hline $\mathrm{T}_{1 / 2}$ (hours) & 1.965 & 1.844 & 2.117 & 1.851 & 1.78 & 1.815 \\
\hline $\mathrm{CL}(\mathrm{mL} / \mathrm{min} / \mathrm{kg})$ & 1.368 & 1.196 & 1.339 & 1.362 & 1.234 & 1.216 \\
\hline
\end{tabular}

$\mathrm{T}_{1 / 2}$ is the time duration to excrete half amount of drug from the body. The order of the excretion is first order in most of the cases.(Zheng et al., 2019) The slope of the graph between concentration and excretion time gives the value of the clearance rate. More the halflife less will be the excretion. A clearance value greater than 5 is high, less than 5 is low.(Kraft et al., 2020; Nanavati \& Mager, 2016) Highest half-life was found for nos86, while the lowest half-life was found for nos83. The highest clearance rate is found for nos107, while the lowest was found for nos41.

\subsubsection{Toxicity properties of top-six noscapines}

Less the toxicity property of a molecule to be the potent drug. During the virtual screening reducing the toxicity property is a prime target for the development of the therapeutic agent.(Gadaleta et al., 2019; Guan et al., 2019) Various toxicities values like ether-à-go-goRelated Gene (hERG) blockers, human hepatotoxicity (H-HT), ames mutagenicity, skin sensitization, half-maximal lethal dose (LD50), drug-induced liver injury (DILI), etc were analyzed and values were given in Table 11. HERG involved in the mediation of an electrical signal to maintain the activity of the heart. The zero value of HERG blocker indicates nonblocking nature while 1 value indicates blockers.(Hull et al., 2019) The highest values of hERG blocker are found for nos37 while the lowest for nos88 and nos83. Liver involved in the excretion of the administered drugs and provide clearance permission. Hence, most of the drugs leave an adverse effect on it. Human hepatotoxicity is measured in two categories 0 and 1 indicating negative and positive toxic probability.(Akakpo et al., 2018; Navarro et al., 
2018) The Highest H-HT value was found for nos 107 while nos37 had the lowest value. The carcinogenic nature of the compound is tested based on the ames mutagenicity parameters. Value 0 indicate ames negative while value 1 indicates ames positive.(Benfenati et al., 2018) Highest ames positive probability was found for nos 83 while the lowest possibility for nos 41 . Skin sensitization is an auto immunological response produced by the foreign substance. Skin sensitization is measured in two categories 0 and 1. Zero indicates non-sensitizing nature while value 1 indicates sensitizing nature.(Toropova \& Toropov, 2019) The highest skin sensitization value was found for nos 83 while the lowest value is found for nos 86 . Half maximal lethal dose $\left(\mathrm{LD}_{50}\right)$ kills $50 \%$ population of the treated animals. $\mathrm{LD}_{50}$ value, 1-50 $\mathrm{mg} / \mathrm{kg}$ is related to high toxic, $51-500 \mathrm{mg} / \mathrm{kg}$ is related to moderate toxicity and $501-5000$ $\mathrm{mg} / \mathrm{kg}$ is related to low toxicity.(Gadaleta et al., 2019) $\mathrm{LD}_{50}$ toxicity order for top six noscapines was found as nos $86<\operatorname{nos} 107<\operatorname{nos} 88<\operatorname{nos} 83<\operatorname{nos} 41<\operatorname{nos} 37$. Drug-induced liver injury (DILI) value of the drug is responsible for liver failure. Since high lipid-soluble drugs metabolized by the liver. DILI values are recorded in two respect 1 and 0 . Value 1 indicates DILI positive while value 0 indicates DILI negative.(Mullins, Beaulac, \& Sylvia, 2019) The highest DILI positive value was found for nos86 while the lowest positive value was found for nos41. Food and Drug Administration (FDA) recommended maximum daily dose (FDAMDD) values for a drug indicate its applicability towards the FDA recommendations based on the studies of about 1200 drugs.(Ferreira \& Andricopulo, 2019) Category 0 indicates FDAMDD negative while category 1 indicates FDAMDD positive nature.(Liu, Oprea, Ursu, Hasselgren, \& Altman, 2016) The highest FDAMDD value was found for nos 88 while the lowest value is found for nos 37.

Table 11 Toxicity properties of top six noscapines

\begin{tabular}{|l|l|l|l|l|l|l|}
\hline Property & \multicolumn{1}{|c|}{$\mathbf{1 0 7}$} & \multicolumn{1}{|c|}{$\mathbf{3 7}$} & \multicolumn{1}{|c|}{$\mathbf{8 6}$} & \multicolumn{1}{|c|}{} & \multicolumn{1}{|c|}{$\mathbf{4 3}$} & \multicolumn{1}{|c|}{} \\
\hline hERG & 0.688 & 0.76 & 0.69 & 0.686 & 0.686 & 0.748 \\
\hline H-HT & 0.66 & 0.382 & 0.524 & 0.618 & 0.442 & 0.45 \\
\hline Ames Mutagenicity & 0.416 & 0.412 & 0.454 & 0.416 & 0.468 & 0.356 \\
\hline Skin sensitization & 0.191 & 0.187 & 0.143 & 0.191 & 0.201 & 0.194 \\
\hline LD $_{50}(\mathrm{mg} / \mathrm{kg})$ & 700.78 & 343.71 & 870.50 & 636.18 & 536.57 & 410.79 \\
\hline DILI & 0.536 & 0.468 & 0.842 & 0.536 & 0.73 & 0.436 \\
\hline FDAMDD & 0.3 & 0.272 & 0.344 & 0.37 & 0.334 & 0.328 \\
\hline
\end{tabular}

\section{Conclusion}


Noscapines have great medicinal importance and its derivatives can be used against the various biological conditions. Based on the molecule docking studies it was found that most of the noscapines have more negative binding energy than the 14 reported drugs. The minimum binding energy of noscapines indicates its stability towards the inhibition of the activity of the protease of SARS-CoV-2. The top-six potential noscapines are nos107, nos37, nos 86, nos 88, nos83 and nos 41 respectively. The active cavity is composed of HIS-41, GLY143, CYS-145, GLU-166, ASN-142, HIS-164, MET-165, GLU-166, GLN-189, MET-49 and THR-199 amino acids. All top six noscapines targeted the same active cavity. ADMET analysis of top-six noscapines shows that they have low toxicity value low excretion properties, moderate metabolic property, high plasma protein binding affinity and moderate absorption properties. Nos107 and nos86 are the two most promising candidates to inhibit protease of SARS-CoV-2. 


\section{References}

Akakpo, J. Y., Ramachandran, A., Kandel, S. E., Ni, H. M., Kumer, S. C., Rumack, B. H., et al. (2018). 4-Methylpyrazole protects against acetaminophen hepatotoxicity in mice and in primary human hepatocytes. Human \& Experimental Toxicology, 37(12), $1310-1322$.

Alexander, G. C., \& Qato, D. M. (2020). Ensuring Access to Medications in the US During the COVID-19 Pandemic. JAMA.

Alijanvand, S. H., Christensen, M. H., Christiansen, G., Harikandei, K. B., Salehi, P., Schiott, B., et al. (2020). Novel noscapine derivatives stabilize the native state of insulin against fibrillation. Int J Biol Macromol, 147, 98-108.

Altinoz, M. A., Topcu, G., Hacimuftuoglu, A., Ozpinar, A., Ozpinar, A., Hacker, E., et al. (2019). Noscapine, a Non-addictive Opioid and Microtubule-Inhibitor in Potential Treatment of Glioblastoma. Neurochem Res, 44(8), 1796-1806.

Auerbach, J., \& Miller, B. F. (2020). COVID-19 Exposes the Cracks in Our Already Fragile Mental Health System. Am J Public Health, e1-e2.

Aydemir, D., \& Ulusu, N. N. (2020). Commentary: Challenges for PhD students during COVID-19 pandemic: Turning crisis into an opportunity. Biochem Mol Biol Educ.

Azam, M. A., \& Jupudi, S. (2019). Structure-based virtual screening to identify inhibitors against Staphylococcus aureus MurD enzyme. Structural Chemistry, 30(6), 21232133.

Bayefsky, M. J., Bartz, D., \& Watson, K. L. (2020). Abortion during the Covid-19 Pandemic - Ensuring Access to an Essential Health Service. $N$ Engl J Med.

Beard, R., Gaboriau, D. C. A., Gee, A. D., \& Tate, E. W. (2019). Chemical biology tools for probing transcytosis at the blood-brain barrier. Chemical Science, 10(46), 1077210778.

Benfenati, E., Golbamaki, A., Raitano, G., Roncaglioni, A., Manganelli, S., Lemke, F., et al. (2018). A large comparison of integrated SAR/QSAR models of the Ames test for mutagenicity(\$). Sar and Qsar in Environmental Research, 29(8), 591-611.

BIOVIA, D. S. (2017). Discovery Studio Modelling Environment, Release 2017.

Bodas, M., \& Peleg, K. (2020). Self-Isolation Compliance In The COVID-19 Era Influenced By Compensation: Findings From A Recent Survey In Israel. Health Aff (Millwood), 101377hlthaff202000382.

Campbell, C. M., \& Kahwash, R. (2020). Will Complement Inhibition be the New Target in Treating COVID-19 Related Systemic Thrombosis? Circulation. 
Celik, S., Albayrak, A. T., Akyuz, S., \& Ozel, A. E. (2019). Synthesis, molecular docking and ADMET study of ionic liquid as anticancer inhibitors of DNA and COX-2, TOPII enzymes. Journal of Biomolecular Structure \& Dynamics, 7, 1-11.

Chakravarty, A. K., Singh, P., \& Kumari, K. (2016). One pot green synthesis of biological potent thiazolopyrans and docking against human pancreatic lipase related protein 1 receptors Inter J Curr Adv Res 5(1), 559-563.

Chandra, R., Madan, J., Singh, P., Chandra, A., Kumar, P., Tomar, V., et al. (2012). Implications of nanoscale based drug delivery systems in delivery and targeting tubulin binding agent, noscapine in cancer cells. Curr Drug Metab, 13(10), 14761483.

Chem3D http://www.cambridesoft.com.

Clarke, A. L., Stephens, A. F., Liao, S., Byrne, T. J., \& Gregory, S. D. (2020). Coping with COVID-19: ventilator splitting with differential driving pressures using standard hospital equipment. Anaesthesia.

Daina, A., Michielin, O., \& Zoete, V. (2017). SwissADME: a free web tool to evaluate pharmacokinetics, drug-likeness and medicinal chemistry friendliness of small molecules. Sci Rep, 7, 42717.

Di, L., Kerns, E. H., \& Carter, G. T. (2009). Drug-Like Property Concepts in Pharmaceutical Design. Current Pharmaceutical Design, 15(19), 2184-2194.

Feinberg, E., Pande, V., \& Cheng, A. (2019). Comparison of random forest and deep learning approaches for ADMET endpoint prediction. Abstracts of Papers of the American Chemical Society, 257.

Feldmann, M., Asselin, M. C., Wang, S., McMahon, A., Hinz, R., Duncan, J., et al. (2014). P-glycoprotein (Pgp) overactivity in pharmacoresistant epilepsy patients with focal cortical dysplasia compared to healthy controls measured using (R)-[11C] verapamil PET and the Pgp inhibitor Tariquidar. European Journal of Neurology, 21, 229-229.

Ferreira, L. L. G., \& Andricopulo, A. D. (2019). ADMET modeling approaches in drug discovery. Drug Discovery Today, 24(5), 1157-1165.

Gadaleta, D., Vukovic, K., Toma, C., Lavado, G. J., Karmaus, A. L., Mansouri, K., et al. (2019). SAR and QSAR modeling of a large collection of LD50 rat acute oral toxicity data. Journal of Cheminformatics, 11(1).

Guan, L. F., Yang, H. B., Cai, Y. C., Sun, L. X., Di, P. W., Li, W. H., et al. (2019). ADMETscore - a comprehensive scoring function for evaluation of chemical drug-likeness. Medchemcomm, 10(1), 148-157. 
Hooli, S., \& King, C. (2020). Generalizability of COVID-19 Clinical Prediction Models. Clin Infect Dis.

https://www.who.int/emergencies/diseases/novel-coronavirus-2019.

Hull, C. M., Genge, C. E., Hobbs, Y., Rayani, K., Lin, E., Gunawan, M., et al. (2019). Investigating the utility of adult zebrafish ex vivo whole hearts to pharmacologically screen hERG channel activator compounds. American Journal of PhysiologyRegulatory Integrative and Comparative Physiology, 317(6), R921-R931.

Kocak, C., Kocak, F. E., Ozturk, B., Tekin, G., \& Vatansev, H. (2020). Cytotoxic, antiproliferative and apoptotic effects of noscapine on human estrogen receptor positive (MCF-7) and negative (MDA-MB-231) breast cancer cell lines. Bratisl Lek Listy, 121(1), 43-50.

Kraft, T. E., Richter, W. F., Emrich, T., Knaupp, A., Schuster, M., Wolfert, A., et al. (2020). Heparin chromatography as an in vitro predictor for antibody clearance rate through pinocytosis. Mabs, 12(1).

Kumar, D., Kumari, K., Jayaraj, A., Kumar, V., Kumar, R. V., Dass, S. K., et al. (2020). Understanding the binding affinity of noscapines with protease of SARS-CoV-2 for COVID-19 using MD simulations at different temperatures. J Biomol Struct Dyn, 114.

Kumar, D., Kumari, K., Jayaraj, A., Kumar, V., Singh, P., Chandra, R., et al. (2020). Selective Docking of Pyranooxazoles Against nsP2 of CHIKV Eluted Through Isothermally and Non-Isothermally MD simulations. ChemistrySelect, 5(14), 42104220.

Kumar, D., Kumari, K., Jayaraj, A., \& Singh, P. (2020). Development of a theoretical model for the inhibition of nsP3 protease of Chikungunya virus using pyranooxazoles. $J$ Biomol Struct Dyn, 30(10), 3018-3034.

Kumar, D., Singh, P., Chandra, R., Kumari, K., \& Kumar, M. (2017). Impact of Gemini Surfactants on the stability of Insulin using computational tools J Nanomed Biother 7, $1-5$.

Kumar, D., Singh, P., Jayaraj, A., Kumar, V., Kumari, K., \& Patel, R. (2019a). A Theoretical Model to Study the Interaction of Erythro-Noscapines with nsP3 protease of Chikungunya Virus. Chemistryselect, 4(17), 4892-4900.

Kumar, D., Singh, P., Jayaraj, A., Kumar, V., Kumari, K., \& Patel, R. (2019b). A Theoretical Model to Study the Interaction of Erythro-Noscapines with nsP3 protease of Chikungunya Virus ChemistrySelect 4(17), 4892-4900. 
Li, X. T., Yan, Z. B., Wang, R. T., \& Yu, K. J. (2019). Preoperative Mean Platelet Volume and Platelet Distribution Width Predict Postoperative Sepsis in Patients with Colorectal Cancer. Biomed Research International, 2019.

Lipinski, C. A. (2000). Drug-like properties and the causes of poor solubility and poor permeability. Journal of Pharmacological and Toxicological Methods, 44(1), 235249.

Liu, T., Oprea, T., Ursu, O., Hasselgren, C., \& Altman, R. B. (2016). Estimation of Maximum Recommended Therapeutic Dose Using Predicted Promiscuity and Potency. Cts-Clinical and Translational Science, 9(6), 311-320.

Lule, V. K., Tomar, S. K., Chawla, P., Pophaly, S., Kapila, S., \& Arora, S. (2020). Bioavailability assessment of zinc enriched lactobacillus biomass in a human colon carcinoma cell line (Caco-2). Food Chemistry, 309.

Ma, C. Y., Yang, S. Y., Zhang, H., Xiang, M. L., Huang, Q., \& Wei, Y. Q. (2008). Prediction models of human plasma protein binding rate and oral bioavailability derived by using GA-CG-SVM method. Journal of Pharmaceutical and Biomedical Analysis, 47(4-5), 677-682.

Mather, T. P. S., Marin, B. G., Perez, G. M., Christophers, B., Paiva, M. L., Oliva, R., et al. (2020). Love in the time of COVID-19: negligence in the Nicaraguan response. Lancet Glob Health.

Mullins, C., Beaulac, K., \& Sylvia, L. (2019). Drug-Induced Liver Injury (DILI) With Micafungin: The Importance of Causality Assessment. Annals of Pharmacotherapy.

Muthiah, D., Henshaw, G. K., DeBono, A. J., Capuano, B., Scammells, P. J., \& Callaghan, R. (2019). Overcoming P-Glycoprotein-Mediated Drug Resistance with Noscapine Derivatives. Drug Metab Dispos, 47(2), 164-172.

Nanavati, C., \& Mager, D. E. (2016). Calculated Log D Is Inversely Correlated With Select Camptothecin Clearance and Efficacy in Colon Cancer Xenografts. Journal of Pharmaceutical Sciences, 105(4), 1561-1566.

Navarro, V. J., Avula, B., Wang, Y. H., Barnhart, H., Verma, M., Khan, I., et al. (2018). Quantitation of Catechin Content in Herbal and Dietary Supplements Implicated in Human Hepatotoxicity: A Preliminary Report from the Drug Induced Liver Injury Network (DILIN). Hepatology, 68, 434a-434a.

Peloso, A., Moeckli, B., Oldani, G., Triponez, F., \& Toso, C. (2020). Response of a European surgical department to the COVID-19 crisis. Swiss Med Wkly, 150, w20241. 
Pettersen, E. F., Goddard, T. D., Huang, C. C., Couch, G. S., Greenblatt, D. M., Meng, E. C., et al. (2004). UCSF chimera - A visualization system for exploratory research and analysis. Journal of Computational Chemistry, 25(13), 1605-1612.

Phua, J., Weng, L., Ling, L., Egi, M., Lim, C. M., Divatia, J. V., et al. (2020). Intensive care management of coronavirus disease 2019 (COVID-19): challenges and recommendations. Lancet Respir Med.

Singh, H., Singh, P., Kumari, K., Chandra, A., Dass, S. K., \& Chandra, R. (2013). A review on noscapine, and its impact on heme metabolism. Curr Drug Metab, 14(3), 351-360.

Singh, P., \& Chandra, R. (2012). Noscapines encapsulated Nanopolymers: A Novel drugCarrier System to Target the Tumor cells Inter J Green Nanotech 4(2), 80-92.

Singh, P., Kumar, D., Vishvakarma, V. K., Yadav, P., Jayaraj, A., \& Kumari, K. (2019). Computational approach to study the synthesis of noscapine and potential of stereoisomers against nsP3 protease of CHIKV. Heliyon, 5(12), e02795.

Singh, P., Kumari, K., Awasthi, S. K., \& Chandra, R. (2016). Virtual Screening and Docking Studies of Synthesized Chalcones: Potent Anti-Malarial Drug Inter J Drug Dev Res $8(1), 49-56$.

Singh, P., Kumari, K., \& Chandra, R. (2016a). Green synthesis of Tetrazines and their role as human cytomegalovirus (HCMV) protease inhibitor J Theor Comp Sci 3, 1-5.

Singh, P., Kumari, K., \& Chandra, R. (2016b). Synthesis, Computational \& Docking Studies of Bis-(4-Hydroxycoumarin-3-Yl) Methanes As Potential Inhibitor For Carbonic Anhydrase, Glyceraldehyde-3-Phosphate Dehydrogenase J Pharm App Chem 2, 81101.

Singh, P., Singh, H., Chandra, A., Dass, S. K., \& Chandra, R. (2013). How Noscapine metabolise Heme? . J Adv Biology 2(1), 77-91.

Singh, P., Vishvakarma, V. K., Pant, B., Yadav, S., Aslam, M., Yadav, J., et al. (2017). Computational docking studies of Noscapines: A potential bioactive agent Amer $J$ Pharmacol Pharmacother, 4(1), 9-14.

Tomlinson, S. M., Malmstrom, R. D., Russo, A., Mueller, N., Pang, Y. P., \& Watowich, S. J. (2009). Structure-based discovery of dengue virus protease inhibitors. Antiviral Research, 82(3), 110-114.

Toropova, A. P., \& Toropov, A. A. (2019). Hybrid optimal descriptors as a tool to predict skin sensitization in accordance to OECD principles (vol 275, pg 57, 2017). Toxicology Letters, 313, 206-206. 
Vincent, J. L., \& Taccone, F. S. (2020). Understanding pathways to death in patients with COVID-19. Lancet Respir Med.

Vishvakarma, V. K., Kumari, K., Patel, R., Dixit, V. S., Singh, P., Mehrotra, G. K., et al. (2015). Theoretical model to investigate the alkyl chain and anion dependent interactions of gemini surfactant with bovine serum albumin. Spectrochim Acta A Mol Biomol Spectrosc, 143, 319-323.

Vishvakarma, V. K., Kumari, K., \& Singh, P. (2020). A Model To Study The Inhibition Of Arginase II With Noscapine \& Its Derivatives. J Pro Res Bioinf 2(1), 1-14.

Vishvakarma, V. K., Patel, R., Kumari, K., \& Singh, P. (2017). Interaction between Bovine Serum Albumin and Gemini Surfactants using Molecular Docking Characterization Inf Sci Lett, 3, 1-9.

Vishvakarma, V. K., Shukla, N., Reetu, Kumari, K., Patel, R., \& Singh, P. (2019). A model to study the inhibition of nsP2B-nsP3 protease of dengue virus with imidazole, oxazole, triazole thiadiazole, and thiazolidine based scaffolds. Heliyon, 5(8), e02124.

Vishvakarma, V. K., Singh, P., Kumar, V., Kumari, K., Patel, R., \& Chandra, R. (2019). Pyrrolothiazolones as Potential Inhibitors for the nsP2B-nsP3 Protease of Dengue Virus and Their Mechanism of Synthesis ChemistrySelect 4(32), 9410-9419.

Vishvakarma, V. K., Singh, P., Kumari, K., \& Chandra, R. (2017). Rational Design of Threo as Well Erythro Noscapines, an Anticancer Drug: A Molecular Docking and Molecular Dynamic Approach Biochemistry \& Pharmacology 6(3), 1-7.

Vishvakarma, V. K. S., N.; Reetu; Kumari, K.; Patel R.; Singh, P. (2019). A model to study the inhibition of nsP2B-nsP3 protease of dengue virus with imidazole, oxazole, triazole thiadiazole, and thiazolidine based scaffolds. Heliyon, 5, e02124.

Vora, J., Patel, S., Sinha, S., Sharma, S., Srivastava, A., Chhabria, M., et al. (2019). Structure based virtual screening, 3D-QSAR, molecular dynamics and ADMET studies for selection of natural inhibitors against structural and non-structural targets of Chikungunya. Journal of Biomolecular Structure \& Dynamics, 37(12), 3150-3161.

Wu, Z. X., Lei, T. L., Shen, C., Wang, Z., Cao, D. S., \& Hou, T. J. (2019). ADMET Evaluation in Drug Discovery. 19. Reliable Prediction of Human Cytochrome P450 Inhibition Using Artificial Intelligence Approaches. Journal of Chemical Information and Modeling, 59(11), 4587-4601.

Xu, C., \& Desta, Z. (2013). In Vitro Analysis and Quantitative Prediction of Efavirenz Inhibition of Eight Cytochrome P450 (CYP) Enzymes: Major Effects on CYPs 2B6, 2C8, 2C9 and 2C19. Drug Metabolism and Pharmacokinetics, 28(4), 362-371. 
Yang, J. M., \& Chen, C. C. (2004). GEMDOCK: A generic evolutionary method for molecular docking. Proteins-Structure Function and Bioinformatics, 55(2), 288-304.

Zheng, L. Q., Yang, S. R., Lan, J. G., Gyr, L., Goubert, G., Qian, H., et al. (2019). Solution Phase and Surface Photoisomerization of a Hydrazone Switch with a Long Thermal Half-Life. Journal of the American Chemical Society, 141(44), 17637-17645. 